\title{
Flujometría por difusión termal para la medida del flujo sanguíneo cerebral regional en la cirugía de los aneurismas cerebrales
}

\author{
F. Verdú-López; J.M. González-Darder; P. González-López y L. Botella Macia
}

Servicio de Neurocirugía. Hospital Clínico Universitario de Valencia. Servicio Valenciano de Salud. Valencia.

Resumen

Introducción. La flujometría por difusión termal (FDT) es una técnica que permite, mediante la implantación de una microsonda en una región cerebral de interés, determinar el flujo sanguíneo cerebral regional (FSCr) y su monitorización cuantitativa $(\mathrm{ml} / 100 \mathrm{~g} / \mathrm{min})$, continua y en tiempo real. El objetivo de nuestro trabajo es mostrar los detalles técnicos y resultados preliminares de esta técnica de monitorización durante la cirugía y el postoperatorio del tratamiento microquirúrgico de los aneurismas cerebrales, donde tendría valor para detectar y cuantificar fenómenos isquémicos relacionados con el clipaje temporal arterial o malposición del clip definitivo.

Material clínico. Han sido monitorizados cinco pacientes ( 4 mujeres y 1 hombre; edad media de 50.8 años), dos de ellos con aneurismas de arteria cerebral media, otros dos con sendos aneurismas de arteria comunicante posterior y coroidea anterior y uno con aneurisma de carótida interna paraclinoideo. Los pacientes fueron intervenidos con técnica microquirúrgica y clipaje de los aneurismas a través de un abordaje pterional. Se utilizó monitorización neurofisiológica peroperatoria y flujometría con microdoppler. Inmediatamente antes de la craneotomía se procedió a la colocación de la microsonda de FDT a través de un minitrépano sobre la línea coronal, en territorio de la arteria cerebral anterior ( $2 \mathrm{~cm}$ de línea media) o de la cerebral media (6 cm de línea media) y en sustancia blanca, a unos 2.5 $\mathrm{cm}$ de profundidad. Los pacientes fueron monitorizados durante la cirugía y el tiempo en que estuvieron en la Unidad de Reanimación. Se realizaron un total de $\mathbf{1 4}$ clipajes temporales (rango 2-4) con un tiempo medio de clipaje de $7.2 \mathrm{~min}$ (rango 1.6-16) y 16 (rango 2-8) recolocaciones de los clips definitivos. En el aneurisma paraclinoideo se utilizó la técnica de aspiración retrógrada manteniendo cerrada la carótida interna $45 \mathrm{~min}$. Se describen casos ilustrativos con diferentes registros

Recibido: 7-05-10. Aceptado: demostrativos.

Conclusiones. El uso de la FDT permite una valoración cuantitativa y en tiempo real del FSCr de las áreas cerebrales de interés monitorizadas durante el tratamiento microquirúrgico de los aneurismas cerebrales, lo que hace posible detectar fenómenos isquémicos y evitar que se produzcan déficits neurológicos. La detección precoz de la isquemia permitiría aplicar medidas terapéuticas más precozmente y con mayor eficacia.

PALABRAS CLAVE: Aneurisma. Flujo sanguíneo cerebral. Flujometría mediante difusión termal. Isquemia. Monitorización intraoperatoria. Oclusión temporal arterial.

Using thermal diffusion flowmetry in the assesment of regional cerebral blood flow in cerebral aneurysm microsurgery

\section{Summary}

Introduction. The thermal diffusion flowmetry (TDF) is a technique that allows the measurement of the regional cerebral blood flow (rCBF) through an implanted microprobe in a cerebral region of interest. The monitoring is continuous, real-time and quantitative $(\mathrm{ml} / 100 \mathrm{~g} / \mathrm{min})$. The purpose of our clinical work has been to show the technical details and preliminary results by using this monitoring technique during the microsurgical management of cerebral aneurysms and along the postoperative period. The aim of the monitoring of the $\mathrm{rCBF}$ is to identify and evaluate ischemic events related with the temporary artery clipping or

Abreviaturas. ACA: arteria cerebral anterior. ACI: arteria carótida interna. ACM: arteria cerebral media. AComA: arteria comunicante anterior. AComP: arteria comunicante posterior. ACorA: arteria coroidea anterior. CTA: clipaje temporal arterial. FDT: flujometría por difusión termal. FSC: flujo sanguíneo cerebral. FSCr: flujo sanguíneo cerebral regional. PEM: potenciales evocados motores. PESS: potenciales evocados somato-sensoriales 
malposition of the final clip.

Clinical materials. A total of five patients have been monitored (4 woman and one man with an average age of 50.8 years). Two patients harboured one aneurysm in the middle cerebral artery, other two patients had two aneurysms each one on the internal carotid artery in the exit of the posterior communicating and anterior choroidal artery and the fifth harboured a paraclinoid internal carotid artery aneurysm. All patients were operated on using standard microsurgical techniques through a pterional approach. Surgery was done under neurophysiological monitoring and direct microdoppler fluometry assesment. Just before craniotomy the TDF microprobe was inserted $2,5 \mathrm{~cm}$ deep into the white matter through a small burr-hole placed on the coronal line and $2 \mathrm{~cm}$ away the midline to measure in the anterior cereral artery vascular sector and $6 \mathrm{~cm}$ away of the midline to measure in the middle cerebral artery territory. Patients were under continuous monitoring during surgery and along the postoperative period in the recovery unit. A total of $\mathbf{1 4}$ temporary artery clippings (between 2-4) with an average total clipping time of 7.2 minutes (ranging 1.6 to 16) and 16 definitive clip replacements (ranging 2 to 8 ) were done at surgery. Patient with paraclinoid aneurysm was operated on using the retrograde aspiration technique and the internal carotid artery was kept closed 45 mimutes. keeping Some illustrative cases and demonstrative records are presented.

Conclusions. The use of TDF allows a quantitative real-time measurement of the $\mathrm{rCBF}$ in the areas of interest monitored during the microsurgical management of the cerebral aneurysms which leads to detect ischemic events helpping to avoid ischemic sequelae. The detection of ischemic events in real time would make possible the use of therapeutic measures ealier and more efficienty.

KEY WORDS: Aneurysm. Cerebral blood flow. Thermal diffusion flowmetry. Intraoperative monitoring. Ischemia. Temporary arterial occlusion.

\section{Introducción}

La causa más importante de morbi-mortalidad aguda tras el tratamiento microquirúrgico de los aneurismas cerebrales rotos y no rotos es la isquemia cerebral. El clipaje temporal arterial (CTA) durante la cirugía de los aneurismas cerebrales es una maniobra útil y a veces imprescindible para alcanzar con éxito la exclusión del aneurisma o controlar la ruptura intraoperatoria del aneurisma ${ }^{7,35}$. Sin embargo, esta acción puede llevar a una hipoperfusión en el territorio vascular correspondiente a la arteria clipada que puede desencadenar fenómenos isquémicos con posibilidad de déficits neurológicos asociados ${ }^{19,21}$. El riesgo de que esto último suceda depende de la intensidad y de la duración de la isquemia en el territorio de interés y esto puede variar mucho en función de la circulación colateral. Otra causa potencial de isquemia y déficit neurológico es la inadecuada colocación del clip definitivo con obstrucción parcial o total del flujo a través de la arteria que soporta el aneurisma.

La flujometría cerebral por difusión termal (FDT) en el territorio vascular de interés permite determinar el flujo sanguíneo cerebral regional (FSCr) en tiempo real de una manera continua y cuantitativa en unidades absolutas $(\mathrm{ml} / 100 \mathrm{~g} / \mathrm{min})$. Esta técnica de monitorización ha sido validada $^{39} \mathrm{y}$ ha sido utilizada en diversas situaciones neuroquirúrgicas ${ }^{15,17,22,23,37,38}$, incluyendo la cirugía de los aneurismas cerebrales $^{36}$. En el presente estudio presentamos nuestra experiencia preliminar de la monitorización peroperatoria del FSCr con FDT en el tratamiento microquirúrgico de los aneurismas cerebrales, donde la incorporamos en un protocolo más amplio de monitorización peroperatoria que incluye la monitorización neurofisiológica y de flujometría directa por microdoppler.

\section{Material Clínico}

Casuística. Desde julio de 2009 han sido intervenidos en nuestro servicio cinco pacientes con patología aneurismática cuyo FSCr ha sido monitorizado mediante la técnica de FDT. La serie incluye cuatro mujeres y un hombre, con una edad media de 50.8 años (rango 42-61). Dos pacientes sufrían aneurismas de arteria cerebral media (ACM), uno roto y el otro no roto. Los otros dos pacientes tenían cada uno de ellos dos aneurismas de arteria carótida interna (ACI) en la salida de las arterias comunicante posterior (AComP) y coroidea anterior (AChA), en ambos casos con ruptura de uno de ellos y también en ambos casos con sólo uno de ellos parcialmente embolizado. El último paciente sufría un aneurisma no roto de ACI paraclinoideo. En el momento de la cirugía el GCS era de 15 puntos y todos fueron dados de alta hospitalaria sin déficits neurológicos.

Técnica microquirúrgica. El clipaje fue llevado a cabo a través de un abordaje pterional y técnica microquirúrgica estándar. Durante la disección del cuello del aneurisma y su clipaje se practicaba de forma preventiva el CTA del vaso aferente. En nuestros pacientes se realizaron un total de 14 CTA (rango 2-4) de la ACM o ACI con un tiempo medio de clipaje de 7.2 min (rango 1.6-16) y 16 (rango 28) recolocaciones de los clips definitivos. En la paciente con aneurisma de ACI paraclinoideo se utilizó la técnica de aspiración retrógrada para la clinoidectomía y clipaje, manteniendo la carótida cerrada durante $45 \mathrm{~min}$. Se utiliza de forma rutinaria la monitorización del flujo sanguíneo de 
los vasos de interés y del saco con una sonda microdoppler (Microvascular 20 MHZ Intraoperative Doppler System, Vascular Technology VTI). Todos los aneurismas fueron excluidos de forma completa. Tras el clipaje definitivo del cuello aneurismático se aplica sobre las paredes arteriales una solución diluida de diltiazem. Tras la cirugía se revierte la anestesia de los pacientes en el mismo quirófano o dentro de las primeras cuatro horas en la Unidad de Reanimación.

Protocolo de monitorización neurofisiológica. Nuestro protocolo quirúrgico en la cirugía aneurismática incluye la monitorización neurofisiológica intraoperatoria, con registro de potenciales evocados somatosensoriales (PESS) de nervio mediano y potenciales evocados motores (PEM) (Nihon Koden Neuropack), lo que obliga a una anestesia general sin utilización de relajantes musculares que puedan interferir en el registro. Para la realización de PESS del nervio mediano se estimula éste a nivel de la muñeca y se registra a nivel cortical mediante electrodos subcutáneos que se colocan según el Sistema Internacional 10-20; de esta manera se obtienen respuestas post-anestesia, situado el paciente en postura quirúrgica y a lo largo de toda la intervención así como al final de ésta, lo que permite valorar el estado de la vía sensitiva. Mediante los PEM se evalúa la integridad de la vía corticobulbar y la vía corticoespinal; los electrodos de estimulación se disponen igualmente mediante el Sistema internacional 10-20 y los electrodos de registro se colocan sobre el vientre de los músculos abductor corto pulgar y extensor común dedos en el miembro superior, abductor corto del primer dedo, cuádriceps y tibial anterior en miembro inferior, orbicular oris de la cara y en musculatura de la lengua. Los parámetros de estimulación utilizados son trenes de entre 4 y 7 pulsos (dependiendo de si se estimula la vía bulboespinal o la corticoespinal), con una intensidad de entre 150 y $200 \mathrm{~V}$, con un intervalo libre de estímulo de entre 1 y $4 \mathrm{~ms}$, una frecuencia de $2 \mathrm{~Hz}$ y una duración de tiempo de estímulo de $50 \mu \mathrm{s}$.

Técnica y colocación de sonda de FDT. Se realiza previamente a la craneotomía, en quirófano y con el paciente ya anestesiado. Se coloca la sonda de monitorización (QFlow 500TM Probe, Hemedex Bowman Perfusion Monitor, Codman) mediante trépano de $3,2 \mathrm{~mm}$ en la región del territorio vascular que nos interese, con una distancia a la línea media de aproximadamente $2 \mathrm{~cm}$ para monitorización de territorio de la arteria cerebral anterior (ACA) y de 6 $\mathrm{cm}$ para monitorizar territorio de la arteria cerebral media (ACM) y arteria carótida interna y subcortical a $2,5 \mathrm{~cm}$ de profundidad desde la duramadre (la sonda vienen marcada y medida en $\mathrm{cm}$ que ayudan a calcular su profundidad). Hay que tener en cuenta las variaciones anatómicas de cada individuo mediante estudio de las pruebas de imagen y además siempre hay que evitar las áreas elocuentes. La sonda de monitorización se conecta a un monitor de cabe- cera con una pantalla y que permite el almacenamiento digital del estudio de cada paciente.

\section{Casos ilustrativos}

Caso 1. Mujer de 48 años con un aneurisma no roto de la bifurcación de la ACM derecha descubierto al realizar pruebas de imagen por episodios de cefalea persistente. La exploración neurológica fue normal. Se decidió tratamiento microquirúrgico mediante craneotomía pterional y clipaje. Previamente a la cirugía, en quirófano y con la paciente ya anestesiada se colocó la sonda de monitorización mediante trépano en región frontal derecha parasagital a $6 \mathrm{~cm}$ de línea media, subcortical a $2,5 \mathrm{~cm}$ de profundidad desde la duramadre, en territorio vascular de la ACM. Se realizó monitorización intraoperatoria de PESS y PEM. En el tiempo quirúrgico previo al CTA de la ACM los valores de FSCr se mantuvieron estables siempre por encima de $20 \mathrm{ml} / 100 \mathrm{~g} / \mathrm{min}$. Durante el CTA (Figura 1) que duró 1 minuto y 40 segundos el FSCr disminuyó desde 45 a 20 $\mathrm{ml} / 100 \mathrm{~g} / \mathrm{min}$ y posterior a la retirada del clipaje se produjo un rápido aumento de $\mathrm{FSCr}$ (hasta $75 \mathrm{ml} / 100 \mathrm{~g} / \mathrm{min}$ ) que posteriormente fue descendiendo poco a poco hasta valores normales. Se demostró además durante la cirugía que tras la aplicación tópica de diltiazem diluido (un antagonista del calcio) aparecía un rápido aumento del FSCr. Los PESS y PEM no mostraron cambios significativos ni durante ni al final de la cirugía. Se monitorizó postoperatoriamente a la paciente durante $24 \mathrm{~h}$ en la Unidad de Reanimación siendo los valores de FSCr estables y dentro de la normalidad en todo momento. En el postoperatorio no se identificó ningún déficit neurológico.

Caso 2. Mujer de 42 años que sufrió un episodio de hemorragia subaracnoidea espontánea con cefalea brusca y rigidez de nuca sin déficits neurológicos. Se realizó arteriografía que mostró sendos aneurismas en AComP y AChA derechas más un tercer posible aneurisma en territorio de comunicante anterior (AcomA). Durante la arteriografía se realizó la embolización parcial del aneurisma de AComP y fue imposible realizar embolización del aneurisma de AChA, por lo que se decidió tratamiento quirúrgico de las lesiones. Se procedió a la colocación de la sonda a una distancia de la línea media de $2 \mathrm{~cm}$ en región frontal derecha, sobre el territorio de la ACA. Esta ubicación de la sonda se decidió por la posible existencia de un aneurisma de AComA, que luego no se demostró en la exploración quirúrgica. Se realizó monitorización intraoperatoria de PESS y PEM. Durante el CTA de la arteria carótida interna derecha no aparecieron variaciones significativas en el FSCr (Figura 2). En este caso aparecieron artefactos por movimiento durante la colocación de los retractores cerebrales. Los PESS y PEM no mostraron cambios significativos. No aparecieron fenómenos de disminución de FSCr 


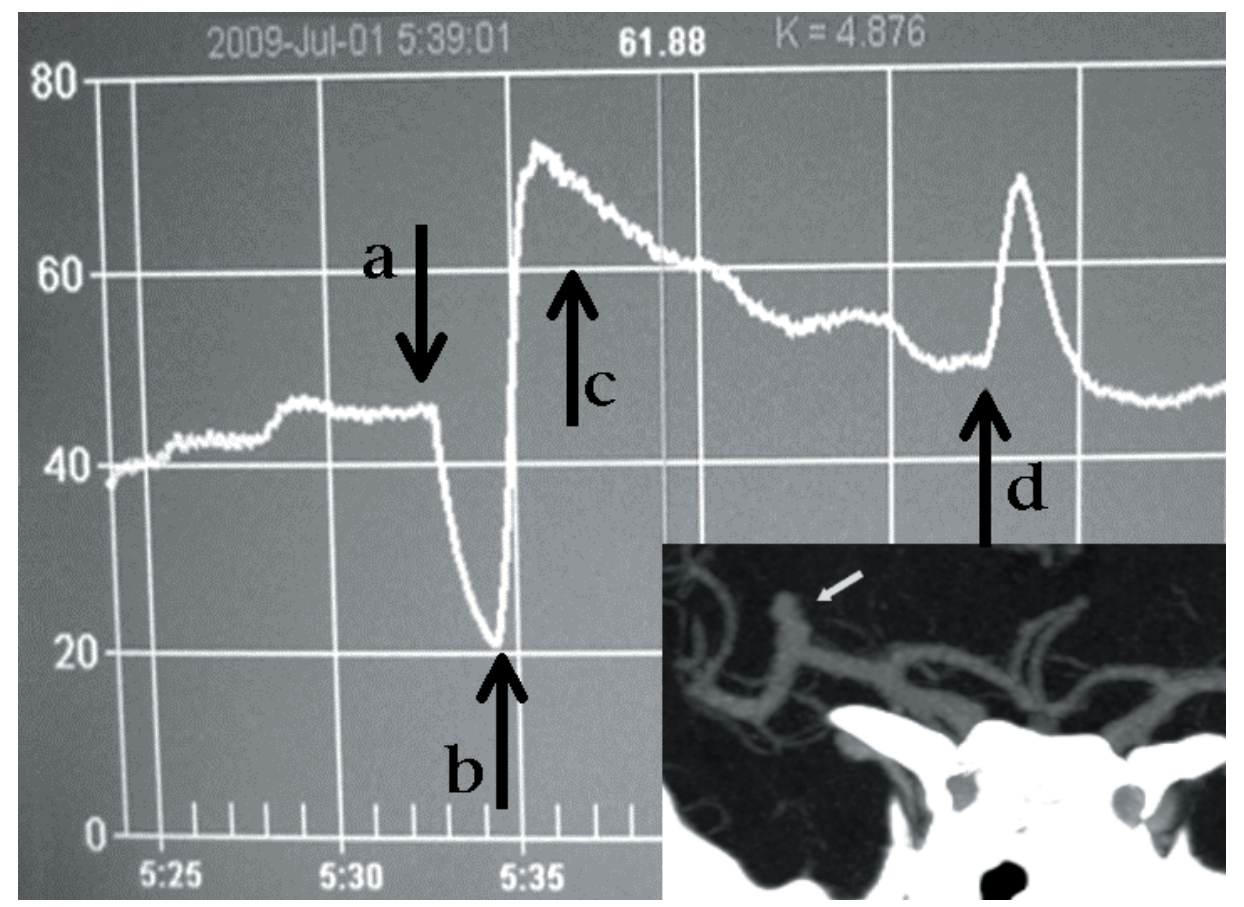

Figura 1. Caso 1. Fotografía tomada de la pantalla del monitor donde se muestra en el eje de abscisas el tiempo y en el eje de ordenadas la perfusión medida en $\mathrm{ml} / 100 \mathrm{~g} / \mathrm{min}$. Valor de $K=4,876$. El clipaje temporal de la arteria cerebral media (a) produce una disminución del FSCr, que se mantiene siempre por encima de $20 \mathrm{ml} / 100 \mathrm{~g} / \mathrm{min}$, seguido de un fenómeno de reperfusión (c) tras la retirada del clip temporal (b). La aplicación tópica de diltiazem diluido sobre la arteria cerebral media produce un rápido aumento del FSCr (d). Se inserta la imagen del aneurisma (flecha) en un estudio de angioTAC.

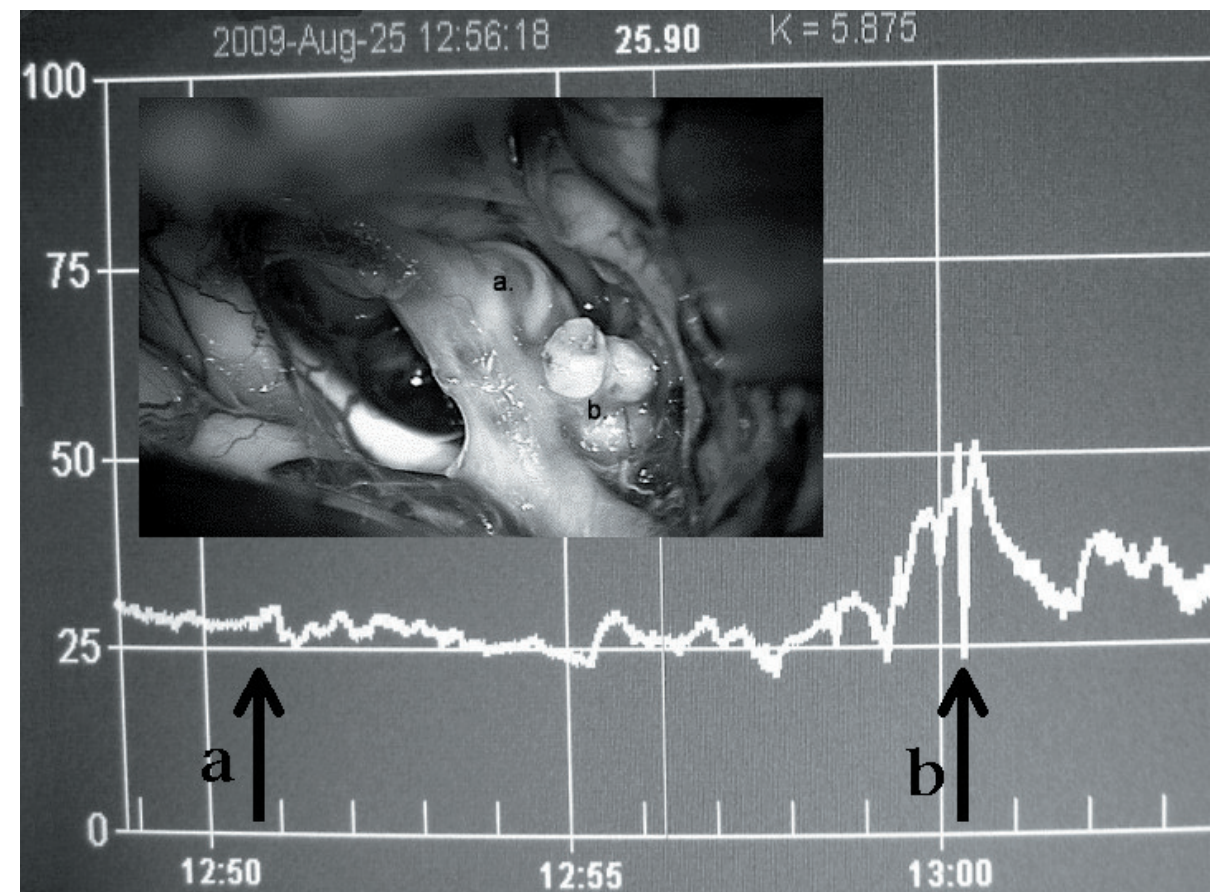

Figura 2. Caso 2. Fotografía tomada de la pantalla del monitor donde se muestra en el eje de abscisas el tiempo y en el eje de ordenadas la perfusión medida en $\mathrm{ml} / 100 \mathrm{~g} / \mathrm{min}$. Valor de $K=5,875$. El clipaje temporal de la arteria carótida interna (a) no produce cambios del FSCr, que se mantiene siempre por encima de $25 \mathrm{ml} / 100 \mathrm{~g} / \mathrm{min}$. Se aprecian variaciones rápidas en el flujo (b), considerados artefactos de medida por movimiento durante la recolocación de los retractores cerebrales. Se inserta una imagen quirúrgica de las lesiones (a: aneurisma de comunicante posterior parcialmente embolizado; b: aneurisma de coroidea anterior). 
durante las 24 primeras horas de postoperatorio ni déficits neurológicos.

\section{Discusión}

El CTA que se realiza durante la cirugía de los aneurismas cerebrales es una maniobra muy interesante para asegurar la correcta exclusión quirúrgica del aneurisma o controlar una ruptura intraoperatoria del aneurisma. El tiempo seguro de oclusión sin que aparezcan déficits neurológicos es enormemente variable según la capacidad vicariante de la circulación colateral para compensar la reducción de flujo impuesta por el CTA ${ }^{19,30}$. Diversos estudios han intentado determinar un tiempo seguro de CTA ${ }^{19,26,30,32}$, que según algunos de ellos puede llegar a ser de 15 minutos. Aún así, usando las medidas de protección que ofrecen los diversos protocolos farmacológicos y anestesiológicos, el daño neurológico aparece aproximadamente entre el 15 y el $20 \%$ de los pacientes s, $^{4,19,25,26,30,35}$. Otro posible riesgo de isquemia peroperatoria por disminución de flujo distal durante la cirugía aneurismática es que debido a una inadecuada colocación del clip definitivo pudiera reducirse el flujo sanguíneo de un territorio vascular al comprometerse la luz del vaso que soporta el aneurisma ${ }^{1}$. Por todo ello es muy importante desarrollar herramientas que permitan durante la cirugía aneurismática detectar lo más precozmente posible situaciones y/o eventos isquémicos para evitar secuelas neurológicas.

Con esta finalidad han sido utilizados múltiples métodos de monitorización durante la cirugía aneurismática cerebral. La monitorización electrofisiológica podría ser adecuada y útil para detectar la isquemia cerebral ${ }^{1,18,21,29,31,33,34}$, aunque ésta puede tener la desventaja del retraso en detectar cambios patológicos ${ }^{4,21}$, insuficiente sensibilidad en detectar isquemia subcortical ${ }^{14}$ y limitaciones anatómicas ${ }^{21,31}$. Aún así la monitorización neurofisiológica constituye una herramienta altamente útil durante la cirugía aneurismática y otras indicaciones de cirugía intracraneal, por lo que nosotros la hemos incluimos en nuestro protocolo. La monitorización neuroquímica mediante diferentes sensores o sondas intracerebrales $\mathrm{o} / \mathrm{y}$ microdiálisis ${ }^{2,13,16,20}$ permite medir la cantidad de diferentes gases en tejido cerebral así como el pH, por lo que estos métodos de monitorización proporcionan información de parámetros que se alteran como consecuencia de la isquemia, precisando siempre una latencia de duración indeterminada. La monitorización ideal requiere de un método que detecte de una manera inmediata las variaciones en el FSCr y por lo tanto la posible isquemia en el tejido de interés antes de que se produzca la lesión funcional o tisular. Esta es la ventaja metodológica que aporta la medida directa del FSCr. Con esta finalidad, antes de la introducción de la FDT se había utilizado la flujometría mediante láser doppler ${ }^{3,10,12}$ y la flujometría mediante difusión termal cortical (sin microsonda subcortical) $)^{5,69,24,25,27}$. Sin embargo, la utilización clínica de estos sistemas se ha visto limitada porque la medición del FSCr se realizaba mediante valores relativos además de las dificultades técnicas que implicaba su implante (pérdida de contacto con la superficie cerebral, la variación de los valores por el contacto con el líquido de irrigación y la inaccesibilidad a regiones alejadas de la craneotomía). Otro sistema utilizado en la cirugía aneurismática es el micro-doppler intraoperatorio que permite mediante contacto de la sonda con el vaso de interés determinar si existe flujo significativo $^{8}$ y que por su facilidad de uso y sencillez resulta una cómoda herramienta. Se ha usado también la arteriografía intraoperatoria, que ha demostrado su utilidad pero a costa de aumentar mucho la complejidad, tiempo y coste quirúr$\operatorname{gicos}^{28}$. Recientemente se ha desarrollado la arteriografía con verde de indocianina ${ }^{11}$, integrada con el microscopio quirúrgico, con buenos resultados preliminares y con mayor facilidad de uso que la arteriografía intraoperatoria, aunque es un método no cuantitativo y que sólo valora la circulación en el campo quirúrgico bajo visión del microscopio.

El sistema de monitorización presentado (QFlow 500TM Probe, Hemedex Bowman Perfusion Monitor, Codman) se basa en la capacidad que poseen los tejidos para transportar calor a través de dos mecanismos físicos: transporte de calor por conducción, que es la transferencia de energía térmica entre dos sistemas por contacto directo de sus partículas sin que exista flujo neto de materia y transporte de calor por convección, que es el calor que se transfiere a través de un fluido en movimiento. La validación de este sistema se ha realizado previamente con buenos y adecuados resultados ${ }^{39}$ y permite la medición del $\mathrm{FSCr}$ en unidades absolutas $(\mathrm{ml} / 100 \mathrm{~g} / \mathrm{min})$ a través del cálculo de perfusión cerebral a nivel capilar, todo ello de forma continua, en tiempo real y junto al paciente. La microsonda se introduce a $2,5 \mathrm{~cm}$ aproximadamente por debajo de la duramadre, en sustancia blanca, y realiza una medición local de un volumen que comprende el de una esfera de alrededor de 4-5 $\mathrm{mm}$ de diámetro. La microsonda presenta dos sensores; un termistor activo distal que incrementa la temperatura basal (aproximadamente dos grados, aunque varía dependiendo de cada situación) y un termistor o sensor pasivo proximal que mide la temperatura basal para compararla con la obtenida posteriormente al calentamiento del termistor distal. El monitor además obtiene la conductividad termal (valor K: que expresa la cualidad de un medio para transmitir calor por conducción $\left[\mathrm{mW} / \mathrm{cm}^{\circ} \mathrm{C}\right]$ ) y con la diferencia de energía se obtiene el FSCr. La ubicación de la sonda dependerá de la región de interés que nos interese monitorizar, con una distancia a la línea media de aproximadamente $2 \mathrm{~cm}$ para monitorización de territorio de ACA y de $6 \mathrm{~cm}$ para monitorizar territorio de $\mathrm{ACM}$ y $\mathrm{ACI}^{36}$, 
contando además con las variaciones anatómicas de cada individuo mediante estudio de la arteriografía y siempre evitando alojarla sobre una área elocuente. El valor K de la sustancia blanca oscila entre 4.8-5.9, valor proporcionado por el aparato durante la calibración y que permite asegurar una óptima medida del FSCr cuando K se encuentra dentro de ese intervalo. El valor K es $>5.9$ cuando la sonda está cerca de un vaso sanguíneo o cuando está situada a mayor profundidad, mientras que en una zona sin flujo sanguíneo el valor de $\mathrm{K}$ es $<4.8$ y si la sonda está fuera de tejido el valor $\mathrm{K}$ es $<1.5$. El valor normal del $\mathrm{FSCr}(\mathrm{ml} / 100 \mathrm{~g} / \mathrm{min})$ en sustancia blanca debe situarse entre $18-25$ y en sustancia gris oscila entre 60-80. Valores indicativos de isquemia o vasoespasmo se situarían por debajo de 15 y valores que podrían indicar infarto estarían por debajo de $10^{37}$. Aunque existe cierta experiencia clínica con la FDT ${ }^{15,17,22,23,36,37,38}$, nuestros resultados en la monitorización subcortical del FSCr durante la cirugía aneurismática son los primeros en ser publicados fuera de los del grupo de trabajo implicado directamente en el diseño y desarrollo de este método de monitorización, por lo que la utilidad clínica real del sistema y su evaluación coste-beneficio deben ser todavía determinados.

Desde un punto de vista técnico y de uso clínico, la FDT sin embargo presenta varias limitaciones e inconvenientes que hay que tener en cuenta. La FDT es una técnica invasiva que precisa efectuar un, aunque mínimo, agujero de trépano con una introducción de una sonda en tejido cerebral con el consiguiente riesgo de lesión que ello conlleva. Además, la medida con FDT es temperatura dependiente y el sistema necesita recalibrarse con cierta frecuencia para que la sonda trabaje adaptándose a las distintas fluctuaciones de temperatura a las que puede verse sometida incluso a pesar de su localización intraparenquimatosa, en las diferentes fases de una cirugía (craneotomía, apertura de dura, irrigación), además de que en una temperatura fuera de un rango comprendido entre $35^{\circ} \mathrm{C}$ y $39^{\circ} \mathrm{C}$ no es capaz de realizar la medición del $\mathrm{FSCr}^{36}$. Otra limitación no menos importante de la FDT es la adecuada elección del territorio vascular donde implantar la sonda ya que variaciones del flujo en un vaso podrían no detectarse si, lógicamente, se está monitorizando otro territorio vascular aunque éste sea adyacente, y por ello es muy importante un adecuado estudio y planificación de cada caso donde quiera utilizarse la FDT. Finalmente, existe además la posibilidad de aparición de artefactos de tipo mecánico como por ejemplo al colocar los retractores cerebrales.

\section{Conclusiones}

El uso de la flujometría por difusión termal permite una valoración cuantitativa y en tiempo real del FSCr para detectar fenómenos isquémicos durante el tratamiento microquirúrgico de los aneurismas cerebrales relacionados habitualmente con el clipaje temporal arterial o colocación inadecuada del clip definitivo. Esto puede ayudar a evitar posibles secuelas neurológicas secundarias a fenómenos isquémicos. La detección de fenómenos de reducción de FSCr en tiempo real permitiría tomar medidas terapéuticas con mayor celeridad y eficacia. En nuestra experiencia, la medida del FSCr con FDT debería ser incluido entre las técnicas de monitorización peroperatoria durante el tratamiento microquirúrgico de los aneurismas cerebrales.

\section{Conflicto de intereses}

Los autores no tienen ningún interés financiero en los productos comerciales citados y utilizados en el estudio.

\section{Bibliografía}

1. Arikan, F., Vilalta, J., Minoves, T., et al.: Detección de episodios de hipoxia tisular isquémica mediante la monitorización neurofisiológica intraoperatoria combinada con la monitorización de la oxigenación tisular en la cirugía aneurismática. Neurocirugía 2008; 19: 113-120.

2. Bachli, H., Langemann, H., Mendelowitsch, A., Alessandri, B., Landolt, H., Gratzl, O.: Microdialytic monitoring during cerebrovascular surgery. Neurol Res 1996; 18: 370376.

3. Boecher-Schwarz, H.G., Ungersboeck, K., Ulrich, P., Mueller-Forell, W., Smolders, D., Perneczky, A.: Pre- and intraoperative methods of controlling cerebral circulation in giant aneurysm surgery. Neurosurg Rev 1995; 18: 85-93.

4. Buchthal, A., Belopavlovic, M., Mooij, J.J.: Evoked potential monitoring and temporary clipping in cerebral aneurysm surgery. Acta Neurochir 1988; 93: 28-36.

5. Carter, L.P., Erspamer, R., White, W.L., Yamagata, S.: Cortical blood flow during craniotomy for aneurysms. Surg Neurol 1982; 17: 204-208.

6. Carter, L.P., White, W.L., Atkinson, J.R.: Regional cortical blood flow at craniotomy. Neurosurgery 1978; 2 : 223-229.

7. Charbel, F.T., Ausman, J.I., Diaz, F.G., Malik, G.M., Dujovny, M., Sanders, J.: Temporary clipping in aneurysm surgery: technique and results. Surg Neurol 1991; 36: 83-90.

8. Charbel F.T, Hoffman W.E, Misra M, Hannigan K, Ausman J.I: Role of a perivascular ultrasonic micro-flow probe in aneurysm surgery. Neurol Med Chir (Tokyo) 1998; (Suppl 38): S35-S38.

9. Choksey, M.S., Chambers, I.R., Jenkins, A., Mendelow, A.D., Sengupta, R.P.: Cortical thermal clearance monitoring in surgery for a giant middle cerebral artery aneurysm. Br J Neurosurg 1993; 7: 673-676.

10. Critchley G.R., O’Neill K.S., Bell, B.A.: Cerebral blood flow and tissue oxygenation monitoring during aneu- 
rysm surgery. Neurol Res 1998; 20 (Suppl 1): S44-S47.

11. de Oliveira, J.G., Beck, J., Seifert, V., Teixeira, M.J., Raabe, A.: Assessment of flow in perforating arteries during intracranial aneurysm surgery using intraoperative near-infrared indocyanine green videoangiography. Neurosurgery 2007; 61(Suppl. 3): 63-72.

12. Fasano, V.A., Urciuoli, R., Bolognese, P., Mostert, M.: Intraoperative use of laser Doppler in the study of cerebral microvascular circulation. Acta Neurochir 1988; 95: 40-48.

13. Hoffman, W.E., Charbel, F.T., Gonzalez-Portillo, G., Ausman, J.I.: Measurement of ischemia by changes in tissue oxygen, carbon dioxide, and pH. Surg Neurol 1999; 51: 654658.

14. Holland, N.R.: Subcortical strokes from intracranial aneurysm surgery: implications for intraoperative neuromonitoring. J Clin Neurophysiol 1998; 15: 439-446.

15. Horn, P., Vajkoczy, P., Thomé, C., Muench, E., Schilling, L., Schmiedek, P.: Xenon-Induced Flow Activation in Patients with Cerebral Insult Who Undergo Xenon-Enhanced CT Blood Flow Studies. American Journal of Neuroradiology 2001; 22: 1543-1549.

16. Hutchinson, P.J., Al-Rawi, P.G., O’Connell, M.T., et al.: Monitoring of brain metabolism during aneurysm surgery using microdialysis and brain multiparameter sensors. Neurol Res 1999; 21: 352-358.

17. Jaeger, M., Soehle, M., Schuhmann, M.U., Winkler, D., Meixensberger, J.: Correlation of continuously monitored regional cerebral blood flow and brain tissue oxygen. Acta Neurochir 2005; 147: 51-6 discussion 56.

18. Kombos, T., Süss, O.: Neurophysiological basis of direct cortical stimulation and applied neuroanatomy of the motor cortex: a review. Neurosurg Focus 2009; 27 (4): E3.

19. Lavine, S.D., Masri, L.S., Levy, M.L., Giannotta, S.L.: Temporary occlusion of the middle cerebral artery in intracranial aneurysm surgery: time limitation and advantage of brain protection. J Neurosurg 1997; 87: 817-824.

20. Mendelowitsch, A., Langemann, H., Alessandri, B., Kanner, A., Landolt, H., Gratzl, O.: Microdialytic monitoring of the cortex during neurovascular surgery. Acta Neurochir 1996; Suppl 67: 48-52.

21. Mizoi, K., Yoshimoto, T.: Permissible temporary occlusion time in aneurysm surgery as evaluated by evoked potential monitoring. Neurosurgery 1993; 33: 434-440.

22. Muench, E., Horn, P., Bauhuf, C., et al.: Effects of hypervolemia and hypertension on regional cerebral blood flow, intracranial pressure, and brain tissue oxygenation after subarachnoid hemorrhage. Crit Care Med 2007; 35: 18441851.

23. Muench, E., Bauhuf, C., Roth, H., et al.: Effects of positive end-expiratory pressure on regional cerebral blood flow, intracranial pressure, and brain tissue oxygenation. Crit Care Med 2005; 33: 2367-72.

24. Ogata, N., Fournier, J.Y., Imhof, H.G., Yonekawa, Y.:
Thermal diffusion blood flow monitoring during aneurysm surgery. Acta Neurochir 1996; 138: 726-731.

25. Ogawa, A., Sato, H., Sakurai, Y., Yoshimoto, T.: Limitation of temporary vascular occlusion during aneurysm surgery. Study by intraoperative monitoring of cortical blood flow. Surg Neurol 1991; 36: 453-457.

26. Ogilvy, C.S., Carter, B.S., Kaplan, S., Rich, C., Crowell, R.M.: Temporary vessel occlusion for aneurysm surgery: risk factors for stroke in patients protected by induced hypothermia and hypertension and intravenous mannitol administration. J Neurosurg 1996; 84: 785-791.

27. Ohmoto, T., Nagao, S., Mino, S., et al.: Monitoring of cortical blood flow during temporary arterial occlusion in aneurysm surgery by the thermal diffusion method. Neurosurgery $1991 ; 28: 49-55$.

28. Payner, T.D., Horner, T.G., Leipzig, T.J., Scott, J.A., Gilmore, R.L., DeNardo, A.J.: Role of intraoperative angiography in the surgical treatment of cerebral aneurysms. J Neurosurg 1998; 88: 441-448.

29. Sako, K., Nakai, H., Kawata, Y., Takizawa, K., Satho, M., Yonemasu, Y.: Temporary arterial occlusion during anterior communicating or anterior cerebral artery aneurysm operation under tibial nerve somatosensory evoked potential monitoring. Surg Neurol 1998; 49: 316-323.

30. Samson, D., Batjer, H.H., Bowman, G., et al,: A clinical study of the parameters and effects of temporary arterial occlusion in the management of intracranial aneurysms. Neurosurgery $1994 ; 34: 22-29$.

31. Schramm, J., Koht, A., Schmidt, G., Pechstein, U., Taniguchi, M., Fahlbusch, R.: Surgical and electrophysiological observations during clipping of 134 aneurysms with evoked potential monitoring. Neurosurgery 1990; 26: 6170 .

32. Symon, L.: Management of giant intracranial aneurysms. Acta Neurochir 1992; 116: 107-118.

33. Szelényi, A., Langer, D., Beck, J., et al.: Transcranial and direct cortical stimulation for motor evoked potential monitoring in intracerebral aneurysm surgery. Neurophysiol Clin 2007; 37: 391-398.

34. Szelényi, A., Kothbauer, K., de Camargo, A.B., Langer, D., Flamm, E.S., Deletis, V.: Motor evoked potential monitoring during cerebral aneurysm surgery: technical aspects and comparison of transcranial and direct cortical stimulation. Neurosurgery 2005; 57 (4 Suppl): 331-338.

35. Taylor, C.L., Selman, W.R., Kiefer, S.P., Ratcheson, R.A.: Temporary vessel occlusion during intracranial aneurysm repair. Neurosurgery 1996; 39: 893-906.

36. Thomé, C., Vajkoczy, P., Horn, P., Bauhuf, C., Hübner, U., Schmiedek, P.: Continuous monitoring of regional cerebral blood flow during temporary arterial occlusion in aneurysm surgery. J Neurosurg 2001; 95: 402-411.

37. Vajkoczy, P., Horn, P., Thome, C., Munch, E., Schmiedek, P.: Regional cerebral blood flow monitoring in the diag- 
nosis of delayed ischemia following aneurismal subarachnoid hemorrhage. J Neurosurg 2003; 98: 1227-1234.

38. Vajkoczy, P., Horn, P., Bauhuf, C., et al.: Effect of intra-arterial papaverine on regional cerebral bloodfFlow in hemodynamically relevant cerebral vasospasm. Stroke 2001; 32: 498-505.

39. Vajkoczy, P., Roth, H., Horn, P., et al.: Continuous monitoring of regional cerebral blood flow: experimental and clinical validation of a novel thermal diffusion microprobe. $\mathrm{J}$ Neurosurg 2000; 93: 265-274.
Verdú-López, F.; González-Darder, J.M.; GonzálezLópez, P.; Botella Macia, L.: Flujometría por difusión termal para la medida del flujo sanguíneo cerebral regional en la cirugía de los aneurismas cerebrales. Neurocirugía 2010; 21: 373-380.

Correspondencia: Dr. Francisco Verdú López. Servicio de Neurocirugía. Hospital Clínico Universitario. Avda. Blasco Ibáñez, 17. 46010-Valencia franciscoverdu@hotmail.es 